\title{
Emergent superconductivity
}

\author{
This month, a Focus issue highlights recent developments in fundamental research on superconductivity.
}

n $\mathrm{n}$ the classical world, dissipation is important in all motion and transport. For instance, the energy losses associated with electric currents are harnessed in everyday life by devices as mundane as light bulbs and heating elements. Therefore, the idea that currents can flow without losing energy - that some materials can superconduct - is remarkable and counterintuitive. This phenomenon is impossible without quantum mechanics, and simultaneously is one of the few places where quantum effects manifest at macroscopic scales. Undoubtedly, the potential technological advancement that would follow the development of a material that superconducts at room temperature and pressure ensures that this field will remain one of the most vigorous in condensed-matter physics.

In one sense, superconductivity is a well-understood phenomenon. We know that, at some temperature, it becomes energetically favourable for electrons to bind together into Cooper pairs. These composite bosons condense into a charged superfluid where dissipationless current is driven by a gradient of the condensate's collective phase. While this picture was worked out in the 1960s, it later transpired that there is a wealth of more-complicated forms of superconductivity that we still do not understand in full.

The most obvious example is the mechanism by which the electrons form Cooper pairs in high-temperature superconductors, but other issues such as the role of dimensionality, strong interactions between the electrons, and band topology have not yet been fully explored. The unifying idea behind this month's Focus issue is that, in order to understand these exotic forms of superconductivity more fully, it is helpful to look at the states from which they emerge. This is not a new idea in itself, but there have been a number of recent developments that we think are timely for a dedicated discussion.

One piece is a Review Article on the quantum breakdown of superconductivity in two dimensions. This newly coined phrase captures the fact that either a metallic or insulating state may arise as superconductivity weakens, widening previous studies of the superconductor-insulator transition. Benjamin Sacépé, Mikhail Feigel'man and Teunis Klapwijk describe various pathways through which superconductivity disappears due to disorder, or how localization aids the fluctuations of the condensate's phase or order parameter. They also discuss how these ideas can transfer to superconducting devices such as Josephson junction arrays that can serve as a model platform for investigating fundamental effects.

We also have a Perspective that discusses the concept of pre-formed Cooper pairs. The idea here is that breakdown of the phase coherence of the condensate will lead to a situation where Cooper pairs exist, but have not formed a superconducting state. This phenomenology has been reported in a number of contexts, including cuprates and complex oxide heterostructures, although it is somewhat controversial. Jeremy Levy and Ivan Božović discuss the similarities and differences in how superconductivity emerges in these two paradigmatic examples, and speculate about the links between nematicity and pre-formed pairs.

\section{"While the general picture of superconductivity was worked out in the 1960s, there is a wealth of more-complicated forms that we still do not understand in full."}

Another aspect that is strongly represented in contemporary research on superconductivity is how that state emerges from situations where interactions between electrons are important. The recent observation of superconductivity in bilayer graphene where there is a small twist angle between the two layers highlights this. Initially, it seemed that the superconductivity was similar to that of high-temperature cuprates, but more experiments on better devices have shown that the picture is not as straightforward as that. While much more work needs to be done, the time is right for an initial assessment of the new understanding and open questions in that field, and Leon Balents et al. provide this in their Perspective.

Finally, what about superconducting states themselves - what exotic forms of superconductivity can emerge from more mundane ones? When a superconductor is driven into a topological phase, it has protected edge states. These are a manifestation of Majorana fermions particles that are their own antiparticles and which have non-abelian exchange statistics. This last fact means that they could be utilized for quantum computation. That long-term goal is still rather distant, and the current most important research question for this field is to unambiguously identify a Majorana mode. The final piece in the Focus issue is a Perspective by Sergey Frolov, Michael Manfra, and Jay Sau that outlines the status of experiments on semiconductor nanowires that have superconductivity induced in them by the proximity effect and are made topological by a magnetic field. This is currently the most advanced Majorana platform, and the authors highlight how breakthroughs in material science and fabrication have been and will be essential for making progress. They also outline many even more exotic states that could be realized in more sophisticated versions of this platform.

There are, of course, many topics in superconductivity that we have not covered, but which remain exciting. The most obvious is the mechanism by which Cooper pairs form in high-temperature superconductors. Recent advances in understanding pair density waves in the cuprates and the influence of nematic fluctuations in iron-based superconductors show that those communities remain very active. This overlaps with the study of multiband superconductivity, where there can be more than one superconducting instability because multiple Bloch bands cross the Fermi surface. This leads to a myriad of possible symmetry forms for the superconducting order, some of which may be manifested in iron-based superconductors.

We should also mention the ongoing efforts to understand the superconductivity in heavy fermion compounds - those that contain elements such as uranium with electrons in atomic $f$ shells - as this probes how superconductivity interacts with strongly correlated phases. The ongoing mystery of the symmetry of the order parameter in strontium ruthenate is also a big puzzle for the field.

It is clear that we cannot highlight the full range of exciting work that is taking place in the area of superconductivity. But we hope that these four pieces capture a snapshot of status of some of the newer and quickly progressing aspects of this field of physics, one that has been so interesting for so long.

Published online: 7 July 2020

https://doi.org/10.1038/s41567-020-0969-7 